\title{
Biología reproductiva del águila Geranoaetus melanoleucus (Aves: Accipitridae) en Chile central
}

\author{
Breeding biology of the black-chested eagle Geranoaetus melanoleucus \\ (Aves: Accipitridae) in central Chile
}

\author{
EDUARDO F. PAVEZ \\ Unión de Ornitólogos de Chile, Casilla 13.183, Santiago 21, Chile, \\ e-mail: eduardopavez@hotmail.com
}

\begin{abstract}
RESUMEN
Durante dos años (julio 1987-enero 1989) se estudió la biología reproductiva del águila (Geranoaetus melanoleucus) en San Carlos de Apoquindo, en Chile central. El inicio del período reproductivo se evidenció por un aumento del número de vuelos en pareja. Los cortejos incluyeron vuelos sincronizados de la pareja, capturas, transporte de material al nido y cópulas. Los traspasos de presas ocurrieron durante todo el año, aunque fuera del período reproductivo fueron escasos y forzados por la hembra. Se localizaron 4,7 nidos pareja ${ }^{-1}$, lo que está en el límite superior documentado para grandes águilas. Fuera del período reproductivo las águilas visitaron ocasionalmente los nidos. Antes de la postura el macho visitó el nido con mayor frecuencia y por menos tiempo que la hembra. En las tres parejas estudiadas se observó visitas a nidos alternativos, con aporte de material y presas. Se promedió 2,3 huevos postura ${ }^{-1}(\mathrm{DE}=0,5, \mathrm{n}=6)$, incubando macho y hembra, y eclosionando el $100 \%$. A los 25 días de edad comenzaron a emerger las plumas de vuelo de los pollos, a los 35 días comían solos las presas aportadas al nido y a los 59 días abandonaron el nido. Durante la crianza la hembra permaneció más tiempo en el nido que el macho, con una tendencia de los adultos a disminuir el tiempo de permanencia en el nido. El $79 \%$ de los pollos sobrevivió a la crianza, lo que se puede considerar un éxito reproductivo elevado, y que se explicaría por la elevada disponibilidad de presas en el sitio de estudio, particularmente de conejos. Los pollos permanecieron en el territorio paterno 10 meses, lo que coincidió con la siguiente postura de huevos, disminuyendo gradualmente su presencia en el área y aumentando la intolerancia de los adultos hacia sus hijos. Los resultados presentados se corresponden con el patrón descrito en la literatura para otras águilas.
\end{abstract}

Palabras clave: águila, Geranoaetus melanoleucus, conducta, reproducción, Chile central.

\begin{abstract}
The breeding biology of the black-chested eagle (Geranoaetus melanoleucus) was studied during a 2-year period (July 1987-January 1989) in San Carlos de Apoquindo, central Chile. The beginning of the reproductive period was evident by the increased number of couple-flights. Courting behavior included synchronized flights, cooperative capture of prey and transport of nest material, as well as copulation. Prey exchanges between adults occurred throughout the year, although outside the breeding season they were uncommon and forced by the female. There were 4.7 nests/couple, a value that is in the upper limit of what has been reported for large eagles. During the non-breeding season, nests were only occasionally visited by the eagles. Before laying, males visited the nest more frequently than females, but for shorter periods of time. Visits to alternative nests carrying prey and nest material were observed in all three couples. The average clutch size was 2.3 eggs $(S D=0.5, n=6)$, with both males and females incubating, and hatching success was $100 \%$. Twenty five days after hatching, flight feathers started to appear in the wings and tail of chicks; after 35 days, chicks started eating by themselves prey items brought to the nest, and after 59 days they left the nest. While tending, females spent more time in the nest than males, although with a tendency of adults to diminish the time spent in the nest over time. Seventy nine percent of the chicks survived, which can be considered a high reproductive success, explained by the high availability of prey in the study site, mainly European rabbits. The chicks remained in the adult's territory for 10 months and gradually started to reduce their presence in the area. This was coincident with the occurrence of the next laying period and an increased adult intolerance. These data concur with the pattern described in the literature for other eagles.
\end{abstract}

Key words: eagle, Geranoaetus melanoleucus, behavior, breeding, central Chile. 


\section{INTRODUCCIÓN}

El águila (Geranoaetus melanoleucus Vieillot 1819) ocupa ambas vertientes de la Cordillera de los Andes, desde Venezuela a Tierra del Fuego, y el sur de Brasil y norte de Argentina (Brown \& Amadon 1968). En Chile es el accipítrido de mayor talla (Jiménez \& Jaksic 1990), distribuyéndose desde Arica a Tierra del Fuego (Goodall et al. 1951), y ocupando terrenos abiertos, desde el nivel del mar hasta más de $2.200 \mathrm{~m}$ de altitud (Barros 1967).

El poco conocimiento sobre su biología e historia natural, se encuentra descrito en revistas locales y documentos de circulación restringida (Jiménez \& Jaksic 1990). En los últimos años se han realizado en Chile trabajos sobre su ecología trófica (Schlatter et al. 1980, Pavez et al. 1992), ecología conductual (Jiménez \& Jaksic 1989) y una revisión bibliográfica de su historia natural (Jiménez \& Jaksic 1990).

La mayoría de las publicaciones sobre la biología reproductiva del águila contienen poca información o son descriptivas y anecdóticas. Housse $(1926,1945)$ describe nidos en la zona central de Chile, sus huevos e incubación, y aspectos del cuidado parental. Goodall et al. (1951), describen someramente los nidos, la puesta y entregan la morfometría de 21 huevos. Barros (1967) se refiere a la conducta de defensa del nido, número y aspecto de los huevos, y posibilidad de abandono de los huevos por perturbaciones antrópicas. Schoonmaker (1984) realizó observaciones de un nido en Perú durante tres días, totalizando 19 h de observación, describiendo la ubicación y orientación del nido, el pollo, sus vocalizaciones y algunas presas aportadas por los padres. Jiménez \& Jaksic (1989) estudiaron diversos aspectos de la biología de la especie en Chile central. Sin embargo, no se refieren a aspectos reproductivos, entregando datos de tamaño de puesta y nidada en una revisión posterior (Jiménez \& Jaksic 1990). Hiraldo et al. (1995) y DeLucca \& Saggese (1995) determinaron cuantitativamente algunos parámetros reproductivos del águila en el suroeste argentino.

Este trabajo aporta una descripción detallada de la conducta reproductiva de la especie en Chile central y entrega algunos parámetros reproductivos cuantitativos, en ambientes en que la liebre europea (Lepus capensis Linnaeus 1758) y el conejo español (Oryctolagus cuniculus Linnaeus 1758) se han constituido en presas abundantes y muy consumidas por las águilas, y que pueden estar contribuyendo al incremento de sus densidades poblacionales y tasas de reproducción.

\section{MATERIALES Y MÉTODOS}

El estudio se realizó en el fundo San Carlos de Apoquindo (33 24' $\mathrm{S}, 70^{\circ} 28^{\prime} \mathrm{O}$ ), ubicado en un área precordillerana de aproximadamente 1.000 ha, $20 \mathrm{~km}$ al este de la ciudad de Santiago, entre 930 y $2.250 \mathrm{~m}$ de altitud. La topografía del área es accidentada, con cerros de pendiente considerable y quebradas profundas. El clima es mediterráneo, con precipitaciones invernales que alcanzan en promedio los $370 \mathrm{~mm}$ anuales. La vegetación corresponde a un matorral mediterráneo, con representación de espino (Acacia caven) en las zonas bajas y de bosques mixtos de quillay (Quillaja saponaria) y litre (Lithrea caustica) en las quebradas. En las laderas secas dominan el chagual (Puya berteroniana) y el bollén (Kageneckia oblonga), además de cactáceas como el quisco (Echinopsis chilensis). En las zonas más altas destaca el olivillo de la cordillera (Kageneckia angustifolia) y a partir de los 1.900 $m$ de altitud se observa una estepa altoandina.

Entre julio de 1987 y enero de 1989, se observó tres parejas de águilas residentes en el área de estudio y cuyos territorios eran contiguos, totalizando $560 \mathrm{~h}$ de observación, distribuidas en 66 excursiones de un día por semana cada una. Cada día se extendía aproximadamente entre las 08:00 y 18:00 h. El estudio cubrió dos períodos reproductivos sucesivos, los que incluyeron cortejos, acondicionamiento de nidos, postura de huevos, incubación y crianza de los pollos. El período no reproductivo fue aquel en que no se observó conductas directamente asociadas a la reproducción.

Las observaciones se realizaron desde puntos elevados con amplia visión y desde escondites, utilizando binoculares 16 x 50 y un telescopio 2040x. Cada águila fue identificada por el patrón particular de coloración de plumas negras ubicadas en el extremo proximal inferior de las alas. Los sexos se distinguieron por el mayor tamaño de las hembras en relación a los machos (Amadon 1959). Los resultados incluyen la información combinada de las tres parejas estudiadas, durante los dos períodos reproductivos.

\section{RESULTADOS}

\section{Inicio del período reproductivo}

Entre abril y julio (otoño y parte del invierno) no se observó conductas directamente asociadas a la reproducción, con una baja frecuencia de vuelos en pareja comparado con agosto y septiembre, meses en que se inició el período reproductivo. Entre 


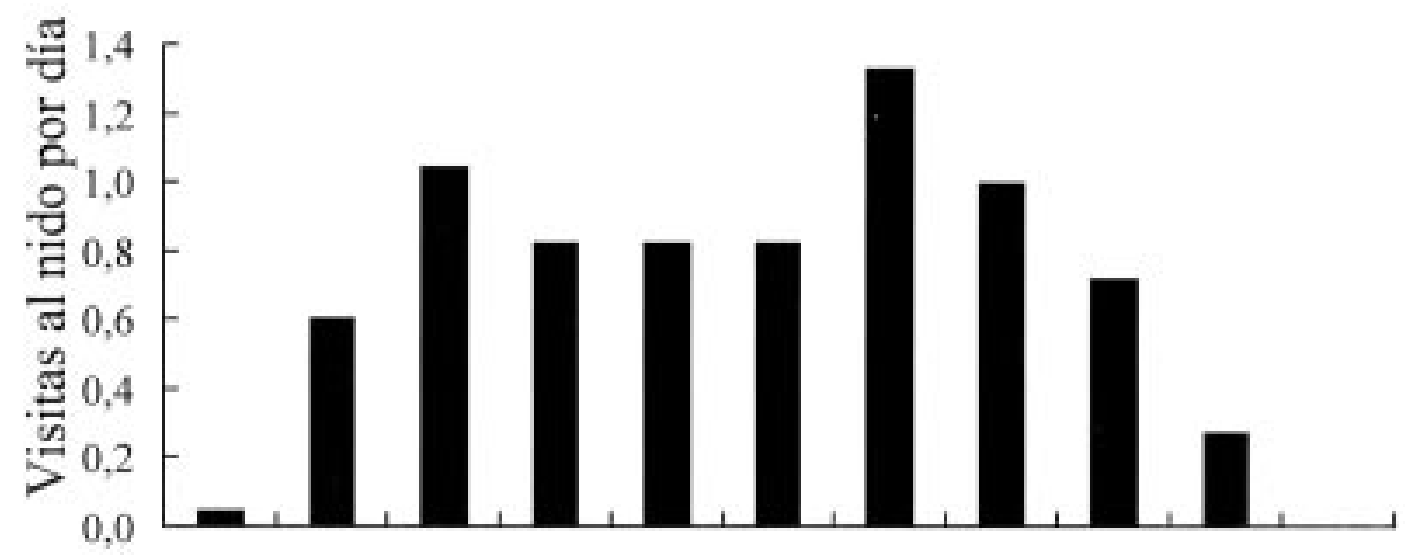

\section{$\begin{array}{llllllllllllllll}8: 00 & 9: 00 & 10: 00 & 11: 00 & 12: 00 & 13: 00 & 14: 00 & 15: 00 & 16: 00 & 17: 00 & 18: 00\end{array}$}

\section{Horas del día}

Fig. 1: Horario de visitas de águilas a los nidos durante el período previo a la incubación en Chile central.

Hourly schedule of visits of eagles to nests previous to the incubation period in central Chile.

abril y julio se registraron 147 avistamientos de águilas adultas residentes, correspondiendo $71 \%$ a águilas en solitario y $29 \%$ a parejas. En cambio, durante agosto y septiembre, de 364 avistamientos, $54 \%$ fueron en solitario y $46 \%$ parejas.

\section{Cortejo}

Entre agosto y septiembre se registró 14 vuelos de cortejo. En 13 de ellos (93\%) la hembra planeaba en círculos o rectilíneamente y el macho la seguía por encima en paralelo. En cuatro ocasiones la hembra también siguió al macho, y en una ocasión sólo la hembra siguió al macho. En siete ocasiones el macho descendió en picado, tres de las cuales fueron siguiendo a la hembra que caía en picado, y en otras dos ocasiones el macho pasó en picado cerca de la hembra mientras esta volaba en círculos. En tres ocasiones el macho se alejó de la hembra en vuelos interpretados como de marcaje territorial, planeando rectilíneo lento hasta el límite de su territorio y regresando junto a la hembra, tomando altura nuevamente en planeos circulares y repitiendo la trayectoria. En una ocasión la hembra lo siguió. Luego de volar juntos la hembra se aperchó en nueve ocasiones, y en siete el macho se aperchó a su lado. En tres ocasiones la hembra y en dos ocasiones el macho, volaron con las patas desplegadas. De los 14 vuelos de cortejo, $14 \%$ fueron inmediatamente sucedidos por cópula, $14 \%$ por capturas de degus (Octodon degus) por parte del macho, y $36 \%$ por transporte de material al nido. Los cortejos se observaron entre las 10:00 y 17:00 h.

\section{Cópulas}

Entre el 1 de septiembre y el 12 de octubre se observaron nueve cópulas, registrándose la primera 33 días después del inicio de los cortejos y 46 días antes de la postura, y con $67 \%$ de ellas siguiendo un patrón definido. Eran precedidas por planeos circulares de la pareja, seguidos por picados de la hembra, la que aterrizaba sobre rocas, arbustos o en el suelo, siendo seguida por el macho, el que aterrizaba encima de ella para copular. En una ocasión la hembra estaba posada mientras el macho volaba en círculos descendiendo sobre ella para la cópula. En otra ocasión la cópula ocurrió luego de aportar material al nido. Una cópula ocurrió en el suelo luego que el macho entregó una presa a la hembra. Durante la cópula la hembra mantenía las alas semiplegadas y el cuerpo horizontal mientras el macho, sobre el dorso de la hembra, batía las alas. Durante las cópulas, que promediaron $9 \mathrm{~s}(\mathrm{DE}=4,2, \mathrm{n}=8)$, ambos emitían vocalizaciones agudas, entrecortadas y de alta intensidad. Las cópulas se observaron regularmente distribuidas entre las 10:30 y 18:00 h.

El $56 \%$ de las cópulas se realizó sobre roca, 33 $\%$ en el suelo, y $11 \%$ sobre arbustos (Puya 
berteroniana). El $22 \%$ se realizó a menos de 15 m del nido y $78 \%$ a más de $150 \mathrm{~m}$. Luego de la cópula, en $67 \%$ de los casos la hembra permaneció posada y el macho voló en picado o permaneció en planeos circulares sobre ella, y en el $33 \%$ restante ambos volaron inmediatamente luego de la cópula.

\section{Traspaso de presas}

Fuera del período reproductivo (abril), en dos ocasiones se observó al macho transportando degus, mientras la hembra lo seguía. En una de estas ocasiones la hembra desistió de la persecución y en la otra el macho comió el degu en vuelo. En mayo, se observó una hembra comiendo un conejo capturado por el macho, no existiendo detalles del traspaso.

Desde agosto se observaron traspasos regulares de presas del macho a la hembra, registrándose 12 hasta la fecha de postura de huevos, a fines de septiembre. En 11 de ellos se avistó al macho transportando presas que incluyeron siete degus, tres conejos y una culebra (Philodryas chamissonis), todas transportadas en las patas, excepto dos degus que fueron sostenidos en el pico. En cinco de los casos el macho transportó la presa hasta el nido; en dos las dejó a menos de 30 m del nido; en tres ocasiones las dejó a más de 150 m del nido; y en una ocasión soltó la presa en vuelo a más de $500 \mathrm{~m}$ del nido, siendo cogida por la hembra en el aire. En uno de los casos el traspaso ocurrió en el sitio de la captura.

En 11 de los traspasos se escuchó vocalizar al macho mientras transportaba la presa y desde el lugar donde llegaba con la presa. Las mismas vocalizaciones fueron emitidas al transportar material al nido. En el traspaso realizado en el aire no hubo vocalizaciones. La hembra acudía al sitio donde el macho llegaba con la presa. En uno de los traspasos la hembra recibió la presa en el aire; en dos ocasiones la hembra llegó al mismo tiempo que el macho al sitio de traspaso; en cinco ocasiones la hembra llegó 30 s después; en dos demoró un minuto; en una demoró dos minutos; y en otra ocasión demoró 3,5 h.

El macho se observaba inquieto al transportar presas, y en 11 de los casos el macho se retiró de inmediato al llegar o acercarse la hembra. En dos de estas ocasiones la hembra cayó violentamente sobre el macho. En otra ocasión el macho soltó la presa en vuelo mientras era seguido por la hembra. En dos oportunidades, fuera del período reproductivo, se observó a la hembra persiguiendo al macho con presa. En seis de los casos la hembra comió en el mismo sitio del traspaso, y en las otras seis ocasiones tomó la presa y la comió a más de $200 \mathrm{~m}$ del sitio del traspaso.

\section{Nido}

Se localizó 14 nidos, distribuidos en seis, cuatro $\mathrm{y}$ cuatro nidos por territorio (promedio $=4,7$ nidos pareja $\mathrm{a}^{-1}$ ), de los cuales $43 \%$ se emplazó en árboles y $57 \%$ en riscos. Todos los árboles usados fueron quillay, siendo la especie arbórea de mayor altura del área. Se ubicaron en árboles de 9 a $11 \mathrm{~m}$ de altura, en un rango de entre 5,5 y 10 m desde la base del árbol, con el nido emplazado cerca del tronco principal. De los ocho nidos en riscos, siete se ubicaron en cornisas y uno en una cueva. Los riscos con nidos ocupados midieron entre 11,4 (con el nido a 4,7 m de altura) y $56 \mathrm{~m}$ de altura (con el nido a $15,7 \mathrm{~m}$ de altura). Una cornisa con nido midió 115 x $211 \mathrm{~cm}$. La cueva con nido midió $105 \mathrm{~cm}$ de profundidad, $186 \mathrm{~cm}$ de ancho, $81 \mathrm{~cm}$ de altura sobre el nido y $61 \mathrm{~cm}$ en la entrada de la cueva. Los nidos estaban formados por ramas secas, y cubiertos en el centro con pastos, formando una capa de $10 \mathrm{~cm}$ de profundidad en uno de ellos. La orientación de los 14 riscos y laderas con nido fue de seis $\mathrm{NO}$, tres $\mathrm{O}$, dos NE, uno SO; uno SE y uno N, y a una altitud de entre 1.200 y $1.800 \mathrm{~m}$.

Fuera del período reproductivo (junio), se registró cuatro arribos de águilas a un nido, tres con aporte de ramas, en dos el macho y en uno la hembra. A principios de agosto comenzaron las visitas regulares a los nidos, reparándolos, usándolos como sitio de traspaso de presas o como posaderos. Desde el momento que se iniciaron los cortejos, 23 días después comenzaron los aportes regulares de material y 73 días después ocurrió la puesta.

Antes de producirse la puesta, en agosto, septiembre y principios de octubre, se totalizaron 18 días de observación, registrándose 135 visitas de águilas a los nidos, de las cuales $41 \%$ fueron del macho, $29 \%$ de la hembra, $24 \%$ de ambos, y en $6 \%$ no se determinó el sexo. El número de visitas por día fue mínimo en agosto, aumentando en septiembre y octubre, con una máxima frecuencia en septiembre (Tabla 1). Las visitas del macho fueron más frecuentes que las de la hembra, especialmente en septiembre, aunque el macho estuvo menos tiempo en el nido, observándose 88 visitas del macho, totalizando 368 min en el nido y promediando 4 min por visita, y 72 visitas de la hembra, completando $707 \mathrm{~min}$ y promediando 10 min por visita.

De los 168 arribos a nidos registrados antes de la puesta, $43 \%$ fueron aportando material, $65 \%$ de las veces por parte del macho y en el $35 \%$ por 
TABLA 1

Visitas de águilas al nido por día durante el período previo a la puesta en Chile central (visitas totales en el mes / días de muestreo en el mes)

Eagle arrivals to nest per day previous to the laying period in central Chile (total monthly visits / monthly sampled days)

\begin{tabular}{llcccc}
\hline Actividad & Sexo & $\begin{array}{c}\text { Agosto } \\
\text { (4 días) }\end{array}$ & $\begin{array}{c}\text { Septiembre } \\
(10 \text { días })\end{array}$ & $\begin{array}{c}\text { Octubre } \\
\text { (4 días })\end{array}$ & $\begin{array}{c}\text { Total } \\
\text { (18 días) }\end{array}$ \\
\hline Visitas sin & Macho & 0,8 & 4,1 & 3,0 & 3,1 \\
objetivo claro & Hembra & 0,5 & 2,7 & 2,8 & 2,2 \\
& Juntos & 1,0 & 2,6 & 0,5 & 1,8 \\
Visitas con & Sexo no determinado & 0,3 & 0,6 & 0,3 & 0,4 \\
aporte de & Macho & 0,5 & 4,0 & 1,0 & 2,6 \\
material & Hembra & 0,5 & 1,8 & 1,0 & 1,3 \\
Visitas para & Sexo no determinado & 0,0 & 0,1 & 0,0 & 0,1 \\
revolcarse & Macho & 0,0 & 1,3 & 1,0 & 0,9 \\
& Hembra & 0,0 & 0,0 & 0,0 & 0,0 \\
Visitas para echarse & & & & 0,4 \\
sin huevos & Macho & 0,0 & 0,3 & 1,3 & 0,4 \\
Visitas & Hembra & 0,0 & 0,3 & 1,0 & 8,8 \\
totales & Macho & 2,3 & 12,3 & 6,8 & 5,7 \\
& Hembra & 2,0 & 7,4 & 5.3 & 0,5 \\
Total de visitas & Sexo no determinado & 0,3 & 0,7 & 0,3 & 15,0 \\
\hline
\end{tabular}

la hembra. La máxima frecuencia de aportes de material fue en septiembre (Tabla 1). Estos fueron en $71 \%$ de los casos ramas secas, en $17 \%$ ramas verdes; y en $12 \%$ pastos secos. Los materiales se transportaron en el pico $65 \%$ de las veces y en las patas $35 \%$, usando las patas para el material más voluminoso. Las ramas verdes se transportaron en el $91 \%$ de los casos en las patas, siendo arrancadas con las patas desde la copa de los árboles. El macho vocalizaba al acercarse al nido con materiales si la hembra estaba en el nido o en sus inmediaciones, de igual modo que cuando llegaba al nido con presas. En el período previo a la puesta se registró actividad en el nido durante todo el día, con máxima frecuencia de arribos entre las 10:00 y 11:00 h y entre las 14:00 y 15:00 h (Fig. 1).

Desde 30 días antes de la puesta, durante nueve días de observación, se registró en 17 ocasiones a las águilas dando forma y profundidad al nido, revolcando y presionando el abdomen y el pecho sobre el nido y rasguñando hacia atrás. Esta conducta se observó sólo en el macho (Tabla 1).

Quince días antes de la puesta las águilas comenzaron a echarse en actitud de incubación, conducta que se intensificó en frecuencia al acercarse la fecha de la postura (Tabla 1). Durante cuatro días de observación se registró 34 arribos al nido, y en 15 ocasiones las águilas se echaron sin huevos. El $53 \%$ de las veces se echó el macho, totalizando $172 \mathrm{~min}$ y promediando $24 \mathrm{~min}$ por vez $(\mathrm{DE}=31,8, \mathrm{n}=7)$, y en $47 \%$ de las veces la hembra, totalizando $231 \mathrm{~min}$ y con un promedio de 38 min por vez $(D E=47, n=6)$.

De los 168 arribos a nidos registrados antes de la puesta, $27 \%$ fueron a nidos no ocupados durante la temporada, $32 \%$ de las veces el macho, $27 \%$ de las veces la hembra, $32 \%$ ambos, y en $9 \%$ de los casos no se determinó el sexo. El $56 \%$ de los arribos a nidos alternativos fue con aporte de material, $7 \%$ con presas, $4 \%$ con revuelco de abdomen y en $33 \%$ sólo se posaron en él.

Se observó dos parejas preparando dos nidos a la vez. Estos eran visitados con igual frecuencia al comienzo del período reproductivo, pero al acercarse la puesta uno era abandonado paulatinamente, mientras que en el definitivo las visitas se incrementaban. El abandono más tardío de un nido alternativo se observó 20 días antes de la puesta. Por los materiales, plumas y fecas encontrados en nidos no ocupados, se concluye que todos los nidos fueron visitados. Dos de las tres parejas ocuparon el mismo nido durante las dos temporadas sucesivas estudiadas. 


\section{Postura e incubación}

Las puestas ocurrieron entre el 28 de septiembre y el 20 de octubre, promediando 2,3 huevos pos$\operatorname{tura}^{-1}(\mathrm{DE}=0,5, \mathrm{n}=6)$ (Tabla 2). Los huevos eran blancos con pequeñas pintas pardo rojizas.

De las 25 observaciones de incubación, $52 \%$ de las veces incubó la hembra y $48 \%$ el macho, promediando la hembra $64 \mathrm{~min}$ por vez $(\mathrm{DE}=$ $48,6, \mathrm{n}=9)$, y el macho $66,8 \mathrm{~min}$ por vez $(\mathrm{DE}=$ $59,9, \mathrm{n}=7)$. Siempre se observó a la hembra pasar la noche en el nido. Al quedar los nidos expuestos al sol, a las horas de mayor calor, las águilas se mantenían de pie dando sombra a los huevos, apoyadas en los tarsos y en la cola, de dorso al sol, con las alas entreabiertas, la cola abierta, las plumas de la cabeza erizadas y jadeando.

Durante el período de incubación se observó al macho aportar todas las presas, las que dejaba en el nido o en sus inmediaciones, siendo consumidas por la hembra fuera del nido. El macho arribaba vocalizando y reemplazaba a la hembra mientras esta comía. Durante este período se observó en una ocasión a la hembra llegar al nido vocalizando con una rama verde en el pico. Aunque no se pudo observar el momento de la eclosión, el período de incubación se estimó en 33 días, eclosionando los 14 huevos puestos (Tabla 2).

\section{Crianza y desarrollo de los pollos}

Los pollos nacieron cubiertos de plumón blanco. A los cuatro días tenían poca actividad, manteniendo casi siempre la cabeza apoyada en el nido. Al arribar un adulto o al moverse éste en el nido, los pollos se arrastraban hacia el adulto, levantando la cabeza en su dirección y apoyados en los tarsos. Los adultos trozaban las presas en el nido y los alimentaban en el pico con pequeños pedazos de carne. Los trozos grandes, con pelos o huesos, o el tracto digestivo de las presas, eran consumidos por los adultos. Durante $23 \mathrm{~min}$ de alimentación, un macho dio 15 trozos a un pollo, 37 al otro, y él comió 12 trozos con pelos o muy grandes. Durante nueve min la hembra dio seis trozos a uno, seis al otro y ella comió cuatro trozos inadecuados para los pollos. En otra ocasión, en 16 min la hembra dio 13 trozos a un pollo, diez al otro, y en tres oportunidades comió intestinos de la presa.

A los 11 días los pollos aun presentaban sólo plumón. El pico y las patas estaban muy desarrollados en relación al cuerpo y levantaban la cabeza observando el entorno. Los pollos intentaban comer solos, pero no eran capaces de desgarrar la carne. Durante las horas de mayor calor, los adultos daban sombra a los pollos de igual modo como fue descrito para los huevos. Cuando la temperatura descendía los adultos dejaban a los pollos solos más tiempo. Los adultos alimentaban a los pollos indistintamente con presas recién aportadas o con las que se acumulaban en el nido.

A los 21 días los pollos comían solos, aunque les era difícil desgarrar la carne. A los 25 días estaban emergiendo las plumas de la cola, espalda y superficie dorsal de las alas. Además, las rémiges secundarias estaban más desarrolladas que las primarias. Vocalizaban al ver a los adultos y caminaban en el nido, aunque estaban casi todo el tiempo apoyados en los tarsos.

A los 35 días los pollos tenían plumas café oscuro en el dorso, rabadilla, abdomen, flancos y en casi todo el dorso de las alas. Aleteaban en el nido, observándose todas las rémiges de escasa longitud, y las rectrices más desarrolladas. En la nuca y muslos tenían escasas plumas. En 10,5 h de observación en dos ocasiones los pollos comieron solos.

TABLA 2

Éxito reproductivo de tres parejas de águilas estudiadas en Chile central durante dos estaciones reproductivas sucesivas

Breeding success of three eagle pairs studied during two successive breeding seasons in central Chile

\begin{tabular}{llcccc}
\hline Año & Actividad & Pareja 1 & Pareja 2 & Pareja 3 & Éxito de nidificación (\%) \\
\hline 1987 & Huevos puestos & 3 & 2 & 2 & 100 \\
& Pollos nacidos & 3 & 2 & 2 & 57 \\
1988 & Pollos salidos del nido & 3 & 2 & 2 & 2 \\
& Huevos puestos & 3 & 2 & 2 & 2 \\
& Pollos nacidos & 3 & 2 & 100 & \\
Éxito de nidificación por pareja (\%) & 50 & 100 & & \\
\hline
\end{tabular}


A los 59 días los pollos se veían totalmente desarrollados, aunque aún presentaban plumón en la parte inferior de las alas y en los flancos. Eran café oscuro dorsalmente, café claro en el pecho, y café profusamente moteado de negro en el abdomen, siendo muy crípticos en vista dorsal y con fondo rocoso. Los pollos hembra eran de talla notoriamente mayor que los machos, con el pecho café más oscuro y más profusamente moteado de negro. A esta edad comenzaron a realizar vuelos cortos en torno al nido. Durante $8 \mathrm{~h}$ de observación el macho adulto aportó cinco presas al nido, retirándose de inmediato y no alimentando a los pollos; la hembra alimentó a los pollos durante 5 min con una presa aportada por el macho. Otro nido observado durante $8 \mathrm{~h}$, con pollos de 60 días, uno aún en el nido y otro en las inmediaciones, no fue visitado por los adultos, y el macho dejó tres presas en las cercanías del nido, de las cuales una fue comida por un pollo y las otras dos por la hembra adulta.

Durante toda la crianza la hembra permaneció mayor tiempo que le macho en el nido, y la tendencia de ambos progenitores fue a disminuir el tiempo de permanencia en el nido al crecer los pollos (Tabla 3). De los 14 pollos nacidos durante las dos temporadas, 11 terminaron su desarrollo en el nido, lo que representa $79 \%$ de éxito de crianza. Tres pollos de un nido, murieron a los 40 días de edad depredados por perros (Canis familiaris) que accedieron al nido ubicado en un roquerío bajo. En ningún momento se observó agresiones entre los pollos.

Durante la crianza, el macho con presas se acercaba al nido vocalizando de igual forma que durante los traspasos de presas en el período de arreglo de nido e incubación. Se observó 17 aportes de presas al nido por parte del macho durante la crianza, de los cuales cuatro fueron traspasadas a la hembra, la que las transportó al nido. La hembra se observó en siete ocasiones aportando presas, de las cuales cinco fueron capturadas por el macho. Las presas aportadas al nido eran más de las que consumían los pollos, por lo que se acumulaban en el nido. Los adultos retiraban las presas antiguas. Durante toda la crianza los adultos aportaron material vegetal verde al nido.

\section{Águilas juveniles}

Los juveniles abandonaron el nido entre fines de diciembre y principios de enero, observándose en el área hasta los 10 meses luego de dejar el nido, momento en que se realizó la siguiente postura, registrándose una baja gradual en la frecuencia de avistamientos de juveniles en el área (Fig. 2). Los juveniles vocalizaban regularmente en el área, en forma aguda e intensa, lo que se interpretó como reclamo de alimento, y que se intensificaban cuando los adultos capturaban o transportaban presas.

Los primeros días luego de abandonar el nido, los juveniles se movían poco en torno al nido. Se observó en dos ocasiones al macho dejando presas junto a los pollos. En lo sucesivo, los juveniles eran cada vez más móviles y agresivos, y los traspasos de presas se basaban más en el acoso de los juveniles hacia los adultos. Cuando los juveniles tenían cuatro meses, se los observó en cuatro ocasiones quitándoles presas a sus padres, no apreciándose resistencia de los adultos. A partir de los cinco meses los juveniles arrebataban las presas a los adultos. Cuando los juveniles tenían entre cinco y nueve meses, se observó nueve disputas de presas a los adultos, en siete de las cuales los adultos vocalizaron amenazando a los juveniles. En dos de las nueve disputas de presas, los adultos agredieron a los juveniles. Durante las disputas los juveniles parecían más prudentes con la hembra que con el macho.

TABLA 3

Actividad de águilas adultas durante el período reproductivo en Chile central

Activity of adult eagles during the breeding period in central Chile

\begin{tabular}{|c|c|c|c|c|c|c|c|}
\hline \multirow{2}{*}{$\begin{array}{l}\text { Edad de } \\
\text { los pollos } \\
\text { (días) }\end{array}$} & \multirow{2}{*}{$\begin{array}{l}\text { Tiempo de } \\
\text { observación } \\
\text { (horas) }\end{array}$} & \multicolumn{2}{|c|}{ Tiempo en el nido (\%) } & \multicolumn{2}{|c|}{$\begin{array}{l}\text { Número de alimentaciones } \\
\text { a pollos }\end{array}$} & \multirow{2}{*}{$\begin{array}{c}\text { Tiempo } \\
\text { alimentando } \\
(\%)\end{array}$} & \multirow{2}{*}{$\begin{array}{l}\text { Tiempo de pollos } \\
\text { con adultos } \\
(\%)\end{array}$} \\
\hline & & Macho & Hembra & Macho & Hembra & & \\
\hline 4 & 6 & 13,0 & 75,6 & 1 & 2 & 13,3 & 88,6 \\
\hline 11 & 8 & 12,9 & 73,7 & 1 & 1 & 6,6 & 74,6 \\
\hline 21 & 10 & 3,3 & 30,9 & 2 & 7 & 15,0 & 32,7 \\
\hline 25 & 7 & 5,7 & 35,1 & 0 & 0 & 0,0 & 40,0 \\
\hline 35 & 10,5 & 0,6 & 18,8 & 1 & 1 & 1,0 & 18,8 \\
\hline 59 & 8 & 0,2 & 1,7 & 0 & 1 & 1,0 & 1,9 \\
\hline
\end{tabular}




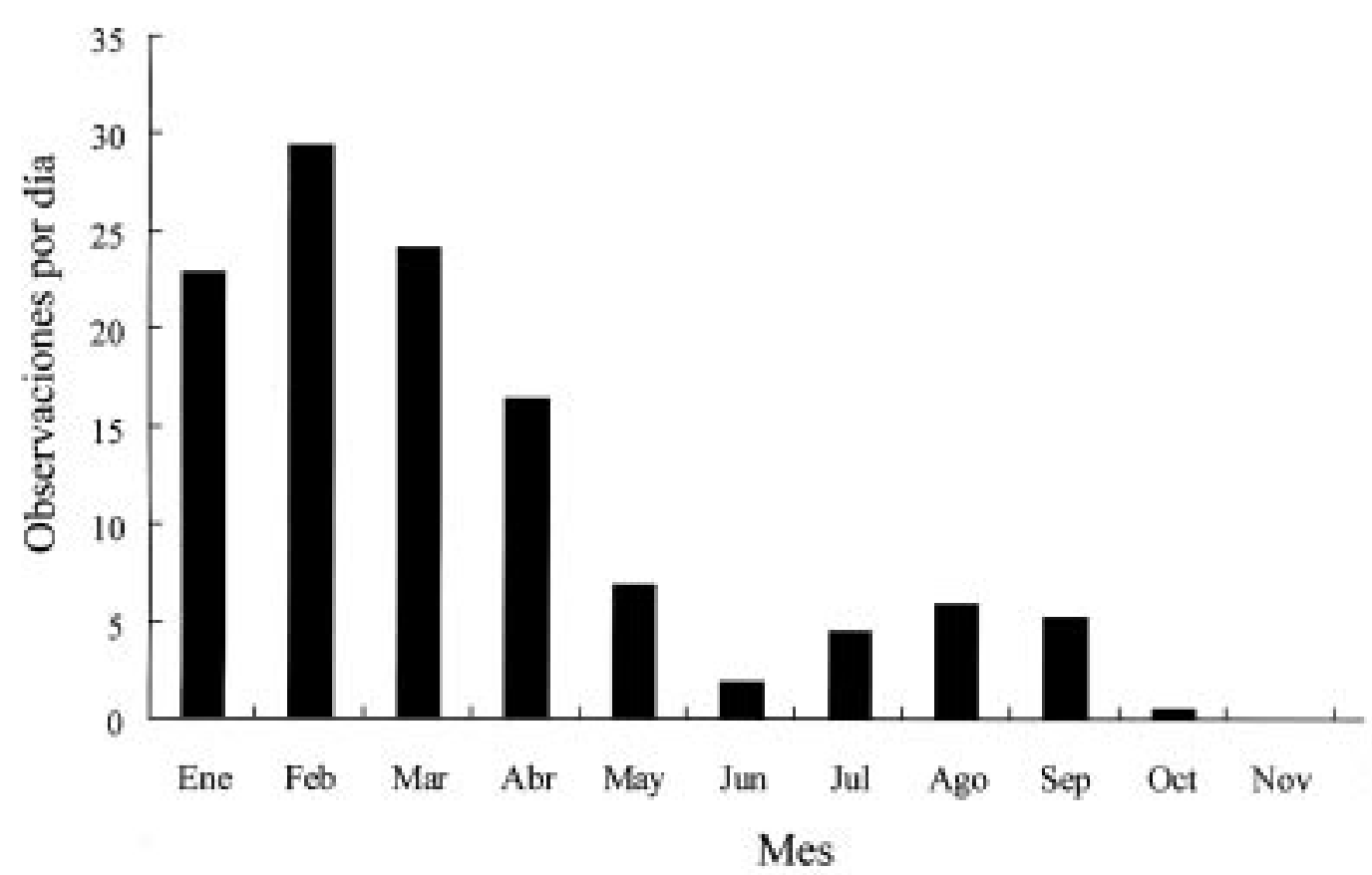

Fig. 2: Número de observaciones de águilas juveniles por día en los territorios paternos durante el año en Chile central.

Number of juvenile eagles per day in the parent's territories throughout the year in central Chile.

Se observó en seis ocasiones agresiones de adultos a juveniles no relacionadas con disputas de alimento, notándose un aumento en la frecuencia e intensidad de las agresiones en la medida que los juveniles eran mayores. A los cuatro meses un juvenil fue amenazado por la hembra mediante vocalizaciones. En dos ocasiones los adultos dieron pasadas suaves en el aire a sus hijos de ocho meses. En octubre se observó tres agresiones violentas de los adultos a sus hijos, y su presencia en el territorio paterno fue mínima, lo cual coincidió con la siguiente puesta de huevos.

Sobre la base de cambios del patrón de coloración de ejemplares juveniles mantenidos en cautiverio (E. Pavez, resultados no publicados), se observó que la coloración definitiva de adulto la adquieren a los cinco años de edad, pudiendo suponerse que a esta edad están maduros sexualmente y en condiciones de establecerse en un territorio. Durante el período de estudio se verificó la muerte de una de las hembras de las parejas estudiadas, lo que representó un $8,3 \%$ de mortalidad de los adultos. La hembra fue rápidamente sustituida por una hembra de cuatro años o subadulta, cuya edad fue determinada por su patrón de coloración, con presencia de algunas plumas café y negras en el abdomen y bajo las alas.

\section{DISCUSIÓN}

Los aspectos de la biología reproductiva del águila aportados en este trabajo se corresponden con el patrón general descrito en la literatura para rapaces de la familia Accipitridae. Concordando con Hiraldo et al. (1995), se observó una ocupación sedentaria del territorio, la que es posible con una adecuada disponibilidad de presas en invierno (Newton 1979). El sedentarismo permite a las aves asegurarse la tenencia de territorio y de pareja y tiene implicancias en la conducta reproductiva, especialmente al inicio y al término del período de reproducción (Newton 1979). En las especies sedentarias, el inicio del período reproductivo se evidencia por un incremento de los vuelos en pareja, especialmente en torno al nido (Newton 1979), hecho que se evidenció en este estudio por un registro de $29,3 \%$ de vuelos en parejas fuera del período reproductivo, contra 45,6\% durante los dos meses previos a la puesta.

El inicio del período de reproducción (agosto), es coincidente en la mayoría de las rapaces de ambientes mediterráneos de Chile central, y estaría determinado por diversos factores como el aumento del fotoperíodo (Lofts \& Murton 1968), el aumento de las temperaturas ambientales (Cavé 1968, Huhtala \& Sulkava 1976), y por una mayor disponibilidad de alimento (Newton 1976, Snyder 
$\&$ Wiley 1976). Este último factor, se asocia a los dos anteriores y se explica por un aumento estacional en la disponibilidad de presas.

Pavez et al. (1992) documentan en la dieta de las mismas águilas y durante el mismo período, $44 \%$ de presas correspondientes a conejos, $19 \%$ a degus, $3 \%$ a aves, $11 \%$ a reptiles y $8 \%$ a insectos. Estos autores observaron un aumento en la abundancia de conejos durante el período reproductivo, con presencia de gazapos en terreno y en la dieta, y un aumento de la diversidad y abundancia de otras presas, tales como aves, reptiles e insectos. En cambio, durante la estación no reproductiva observaron un aumento en la proporción de degus consumidos. Por lo tanto, en el área de estudio existiría una asociación entre el período de reproducción del águila y una mayor disponibilidad de presas, principalmente de conejos, reptiles e insectos.

Los vuelos de cortejo siguieron un patrón definido para Buteo, con planeos circulares sincronizados de la pareja (Fitch et al. 1946, Weir \& Picozzi 1975), además de capturas y traspasos de presas, visitas y transporte de material al nido, cópulas, y marcaje territorial mediante vuelos con un patrón claramente definido, rectilíneos a gran altura sobre el territorio.

Aunque en algunos grupos de Falconiformes, tales como los Circus, los traspasos de presas del macho a la hembra suelen ser aéreos (Jiménez \& Jaksic 1988), en la mayoría de las especies suelen ocurrir en el nido, o en su vecindad. En este trabajo se registraron, durante el período previo a la puesta, $45 \%$ de los traspasos en el nido y $18 \%$ a menos de $30 \mathrm{~m}$, lo que suma un $63 \%$ del total de traspasos.

El nido debe proveer seguridad contra depredadores, existiendo una relación inversa entre el grado de intervención de las áreas, o presencia de enemigos, con el grado de accesibilidad a los nidos (Hickey 1942). La presencia humana es un factor importante que determina la instalación de nidos en sitios inaccesibles (Newton 1979). En el área de estudio los puntos más inaccesibles correspondieron a grandes árboles y acantilados, habiéndose registrado $43 \%$ de los nidos en árboles (los más altos del área) y $57 \%$ en riscos. Casi todos los nidos se ubicaron en sitios inaccesibles para potenciales predadores naturales, los que podrían corresponder en el área al zorro culpeo (Pseudalopex culpaeus) y al quique (Galictis cuja). El único nido ubicado en un pequeño acantilado de fácil acceso por tierra fue depredado por perros. Si se considera el gran número de pollos de águilas robados de nidos que llegan a centros de rehabilitación (E. Pavez resultados no publicados), podría suponerse una ele- vada depredación humana sobre nidos de esta especie en Chile central.

El uso sucesivo de un nido puede determinar su gran tamaño, además de la presencia de viejas estructuras en el suelo (Bent 1938). De hecho, debajo de todos los nidos ubicados en árboles, y de algunos en acantilados, se observó viejas estructuras de nidos, lo que demuestra su uso o reparación sucesiva. La única pareja que cambió de nido de una temporada a otra, fue la que sufrió la pérdida de los pollos durante la segunda temporada.

Los 4,7 nidos pareja ${ }^{-1}$ registrados en el área de estudio se asemejan a los 4,0 nidos pareja ${ }^{-1}$ documentados por Hiraldo et al. (1995) en el suroeste argentino, y está en el límite superior comunicado para otras especies de grandes águilas (Brown 1969, Fasce 1979, Jordano 1981, Mathieu \& Choisy 1982, Fernández \& Leoz 1986). El tener varios nidos por pareja representaría una ventaja para las grandes rapaces, cuyos nidos demandan una costosa construcción, permitiendo una elección de última hora en caso de perturbaciones (Newton 1979). El registro de águilas visitando nidos y aportando material fuera del período reproductivo, tal como fue observado en este estudio, es normal en rapaces sedentarias y podría contribuir al marcaje territorial (Newton 1979).

En el acondicionamiento de nidos el macho fue más activo que la hembra, y esta permaneció más tiempo en el nido, lo que concuerda con la literatura en relación a que las hembras bajan su actividad y acumulan reservas durante el período previo a la puesta (Newton 1979). La conducta de presionar con el abdomen y rasguñar el nido para darle profundidad se observó sólo en el macho, aunque Platt (1977) la describe en machos y hembras de otras especies rapaces. La conducta de echarse en el nido, observada en machos y hembras desde 15 días antes de la puesta, es descrita en las hembras de otras rapaces y permitiría probar el nido frente a posibles perturbaciones que podrían determinar el cambio a un nido alternativo antes de la puesta (Newton 1979).

La variación en la tasa reproductiva de rapaces cazadoras de micromamíferos debido a variaciones en la disponibilidad de alimento se encuentra bien descrita en la literatura (Balfour 1957, Malherbe 1963). El promedio de postura de 2,3 huevos pareja $^{-1}$ observado en este estudio se corresponde con los 2,6 y 2,2 huevos nido ${ }^{-1}$ documentados para la misma especie en el sur de Argentina (DeLucca \& Saggese 1995, Hiraldo et al. 1995) en áreas con abundancia de lagomorfos. Sin embargo, el éxito de crianza de 1,8 pollos nido $^{-1}$ fue superior al 1,1 pollos nido ${ }^{-1}$ registrado por Hiraldo et al. (1995) en Argentina. 
El patrón de turnos observado durante la incubación se ha descrito con frecuencia en rapaces cazadoras, con la hembra incubando de noche y el macho cazando y reemplazándola mientras ella come, pudiendo el macho incubar hasta 1/3 del día (Green 1976, Platt 1977, Newton et al. 1978).

Algunas especies de grandes águilas ponen solo dos huevos, describiéndose como normal el cainismo o muerte de un pollo por agresión de su hermano a temprana edad (Brown 1966, Gargett 1970, 1971, Meyburg 1974). En este estudio los tamaños de nidada fueron elevados (2,3 pollos nido $^{-1}$ ) no registrándose agresiones entre pollos, lo que podría relacionarse con la elevada disponibilidad de alimento observada, pudiendo especularse que podrían haberse manifestado agresiones si el alimento hubiese sido más escaso. En efecto, en Accipitridae de talla media, el cainismo facultativo por estrés alimentario (Simmons 1988) o agresión y muerte entre pollos durante períodos de hambruna se describe como normal (Balfour 1957, Newton 1976). Este ocurriría en cualquier momento de la crianza y en especies con tamaños de nidadas numerosos y variables. Cabe señalar que en los dos nidos con huevos observados por Jiménez \& Jaksic (1990) en Chile, eclosionó un huevo por cada dos, y que en cinco nidos con pichones observados por los mismos autores siempre había un sólo pichón. De Lucca \& Saggese (1995) observaron cinco nidos en el sur de Argentina, en tres de los cuales registraron agresiones entre hermanos y reducción de nidada, a pesar de haber una adecuada disponibilidad de alimento, lo que según estos autores, y basados en una proposición de Simmons (1988), sería un cainismo asociado a las altas densidades poblacionales de rapaces. Lo anterior indica para el águila una tasa de reproducción variable, asociada a la disponibilidad de alimento, y con posible cainismo facultativo.

El patrón de permanencia de los adultos en el nido con los pollos fue esperable, en relación a que la hembra permaneció más tiempo que el macho y a la disminución gradual en el tiempo de permanencia en el nido, lo que según Newton (1979) está relacionado con tres etapas sucesivas: (1) la hembra pasa mucho tiempo echada sobre los pollos para proveerles calor, (2) la hembra cuida a los pollos desde las proximidades del nido mientras el macho caza, y (3) macho y hembra cazan para los pollos.

El tiempo de permanencia de los pollos en el territorio paterno se describe como muy variable dentro y entre especies. Por ejemplo para el aguilucho de cola roja (Buteo jamaicensis) el rango va de 30 a 70 días (Johnson 1973), y para el ratonero común (Buteo buteo) va de 2 a 7 meses (Picozzi \& Weir 1976). En cualquier caso, la permanencia es necesariamente limitada por el inicio de la siguiente temporada reproductiva (Newton 1979), hecho que en nuestro estudio se verificó 10 meses luego de abandonado el nido. La permanencia prolongada en el territorio paterno es posible en aves sedentarias ya que en las especies migratorias el abandono del área de reproducción coincide con la migración de adultos y juveniles. Pavez et al. (1992) describieron para los mismos ejemplares juveniles de este estudio, la frecuente captura de insectos en un principio, asociado al entrenamiento en la caza, ya que su aporte en biomasa es mínimo, por lo que requieren un complemento con presas mayores aportadas por los adultos.

La sustitución como pareja de un ejemplar adulto por otro de cuatro años, es decir subadulto, es común en otras águilas en áreas en que la mortalidad de adultos es alta (Sanderman 1957, Valverde 1960).

\section{AGRADECIMIENTOS}

Agradezco a Fabián Jaksic, Álvaro Palma y especialmente a Jaime Jiménez por sus comentarios y recomendaciones relativas al manuscrito.

\section{LITERATURA CITADA}

AMADON D (1959) The significance of sexual differences in size among birds. Proceedings of the American Philosophical Society 103: 531-536.

BALFOUR E (1957) Observations on the breeding biology of the hen harrier in Orkney. Bird Notes 27: 177-183, 216-224.

BARROS R (1967) Notas sobre el tiuque cordillerano y el águila. Revista Universitaria (Chile) 52: 105-112.

BENT AC (1938) Life histories of North American birds of prey. United States National Museum Bulletin 170: $1-482$.

BROWN L (1966) Observation on some Kenya eagles. Ibis 108: 531-572.

BROWN L (1969) Status and breeding success of golden eagles in north-west Sutherland in 1967. British Birds 62: 345-363.

BROWN L \& D AMADON (1968) Eagles, hawks and falcons of the world. McGraw-Hill Company, New York, New York. 945 pp.

CAVE AJ (1968) The breeding of the kestrel, Falco tinnunculus L., in the reclaimed area Oostelijk Flevoland. Netherlands Journal of Zoology 18: 313 407.

DE LUCCA ER \& MD SAGGESSE (1995) Fratricidio en el águila mora Geranoaetus melanoleucus. Hornero 14: $38-39$. 
FASCE P (1979) Delláquila reale nelle Alpi occidentali italiana e nell Apenino Settentrionale. Revista Italiana d' Ornitologia 2: 34-39.

FERNÁNDEZ C \& J LEOZ (1986) Características de los nidos de águila real (Aquila chrysaetos) en Navarra. Munibe 38: 53-60.

FITCH HS, F SWENSON \& DF TILLOTSON (1946) Behavior and food habits of the red-tailed hawk. Condor 48: 205-237.

GARGETT V (1970) Black eagle experiment II. Bokmakierie 22: 32-35.

GARGETT V (1971) Some observations on black eagles in the Matopos, Rhodesia. Ostrich Supplement 9: 91124.

GOODALL JD, AW JOHNSON \& RA PHILLIPI (1951) Las aves de Chile, su conocimiento y sus costumbres. Volumen II. Platt Establecimientos Gráficos, Buenos Aires, Argentina. 443 pp.

GREEN R (1976) Breeding behaviour of ospreys Pandion haliaetus in Scotland. Ibis 118: 475-490.

HICKEY JJ (1942) Eastern populations of the duck hawk. Auk 59: 176-204.

HIRALDO F, JA DONAZAR, O CEBALLOS, A TRAVAINI, J BUSTAMANTE \& M FUNES (1995) Breeding biology of a grey eagle-buzzard population in Patagonia. Wilson Bulletin 107: 675-685.

HOUSSE R (1926) El águila de Chile. Revista Chilena de Historia Natural 30: 113-121.

HOUSSE R (1945) Las aves de Chile en su clasificación moderna, su vida y sus costumbres. Ediciones de la Universidad de Chile, Santiago, Chile. 390 pp.

HUHTALA K \& S SULKAVA (1976) Breeding biology of the goshawk. Suomen Luonto 6: 299-300.

JIMÉNEZ JE \& FM JAKSIC (1988) Ecology and behavior of South American cinereous harriers, Circus cinereus. Revista Chilena de Historia Natural 61: 199-208.

JIMÉNEZ JE \& FM JAKSIC (1989) Behavioral ecology of gray eagle-buzzards, Geranoaetus melanoleucus, in central Chile. Condor 911: 913-921.

JIMÉNEZ JE \& FM JAKSIC (1990) Historia natural del águila Geranoaetus melanoleucus: una revisión. Hornero 13: 97-110.

JOHNSON SJ (1973) Post-fledging activity of the redtailed hawk. Journal of Raptor Research 7: 43-48.

JORDANO P (1981) Relaciones interespecíficas y coexistencia entre el águila real y el águila perdicera en Sierra Morena Central. Ardeola 28: 67-87.

LOFTS B \& RK MURTON (1968) Photoperiodic and physiological adaptation regulating avian breeding cycles and their ecological significance. Journal of Zoology (London) 155: 327-394.
MALHERBE AP (1963) Notes on birds of prey and some others at Boshoek north of Rustenburg during a rodent plague. Ostrich 34: 95-96.

MATHIEU R \& JP CHOISY (1982) L'Aigle royal (Aquila chrysaetos) dans les Alpes meridionales francaises de 1964 á 1980. Biévre 4: 1-32.

MEYBURG B (1974) Sibling aggression and mortality among nestling eagles. Ibis 116: 224-228.

NEWTON I (1976) Breeding of sparrowhawks (Accipiter nisus) in different environments. Journal of Animal Ecology 45: 831-849.

NEWTON I (1979) Population ecology of raptors. T \& A D Poyser Ltd., Berkhamsted, United Kingdom. 399 pp.

NEWTON I, E MEEK \& B LITTLE (1978) Breeding ecology of the merlin in Northumberland. British Birds 71: 376-398.

PAVEZ EF, CA GONZÁLEZ \& JE JIMÉNEZ (1992) Diet shits of black-chested eagles (Geranoaetus melanoleucus) from native prey to European rabbits in Chile. Journal of Raptor Research 26: 27-32.

PICOZZI N \& DN WEIR (1976) Dispersal and causes of death in buzzards. British Birds 69: 193-201.

PLATT JB (1977) The breeding behaviour of wild and captive gyr falcons in relation to their environment and human disturbance. Tesis de Doctorado (Ph.D.), Cornell University, Ithaca, New York. 173 pp.

SANDERMAN PW (1957) The breeding success of golden eagles in the southern Grampians. Scottish Naturalist 69: 148-152.

SCHLATTER RP, JL Y ÁÑEZ \& FM JAKSIC (1980) Foodniche relationships between Chilean eagles and redbacked buzzards in central Chile. Auk 97: 897-898.

SCHOONMAKER P (1984) Observations on the nesting of the black-chested buzzard-eagle (Geranoaetus melanoleucus) in Peru. Condor 86: 221-222.

SIMMONS R (1988) Offspring quality and the evolution of cainism. Ibis 130: 339-357.

SNYDER NF \& JW WILEY (1976) Sexual size dimorphism in hawks and owls of North America. Ornithological Monographs 20: 1-96.

VALVERDE JA (1960) La population d'aigles imperiaux (Aquila heliaca adalberti) des marismas du Guadalquivir; son évolution depius un siecle. Alauda 28: 2026.

WEIR D \& N PICOZZI (1975) Aspects of social behavior in the buzzard. British Birds 68: 125-141. 